\title{
Asymptotic behaviour of solutions of linear differential equations with delay
}

\author{
by Josef Diblík (Brno)
}

\begin{abstract}
Inequalities for some positive solutions of the linear differential equation with delay $\dot{x}(t)=-c(t) x(t-\tau)$ are obtained. A connection with an auxiliary functional nondifferential equation is used.
\end{abstract}

1. Introduction. In this paper we will obtain inequalities for some solutions of the linear differential equation with delay

$$
\dot{x}(t)=-c(t) x(t-\tau)
$$

where $c: I \rightarrow \mathbb{R}, I=\left[t_{0}, \infty\right), t_{0}=$ const, $c \in C(I)$ and $0<\tau=$ const.

Although the asymptotic properties of solutions of linear differential equations with delay were widely investigated in connection with their numerous applications (e.g. by Bellman and Cooke [1], Hale [3], Kolmanovskij and Nosov [5] and Lakshmikantham and Leela [6]) the considered case is not yet described.

We apply our results to some more common classes of differential equations, e.g. to the equation

$$
\dot{x}(t)=a(t) x(t)-b(t) x(t-\tau)
$$

where the functions $a$ and $b$ are continuous on $I$.

2. The auxiliary results. Now we investigate the asymptotic behaviour at infinity of solutions of the auxiliary functional nondifferential equation of the type

$$
\lambda(t)-\lambda(t-\tau)=\omega(t)
$$

where $\omega \in C(I)$.

1991 Mathematics Subject Classification: Primary 34K25.

Key words and phrases: linear differential equation with delay, asymptotic behaviour. 
If we suppose that on $\left[t_{0}-\tau, t_{0}\right)$ the function $\lambda$ is defined by means of an initial function $g \in C\left(\left[t_{0}-\tau, t_{0}\right)\right)$, i.e.

$$
\lambda(t)=\lambda_{g}(t) \equiv g(t), \quad t \in\left[t_{0}-\tau, t_{0}\right)
$$

then we may define $\lambda(t)$ from (3) stepwise as

$$
\lambda(t)=\lambda_{g}(t) \equiv \lambda_{k}(t) \quad \text { for } t \in\left[t_{0}+(k-1) \tau, t_{0}+k \tau\right)
$$

where $\lambda_{k}(t) \equiv \lambda_{k-1}(t-\tau)+\omega(t)$. In that way we may construct a continuous or continuously differentiable function $\lambda_{g}$ on $\left[t_{0}-\tau, \infty\right)$ which satisfies (3) on $I$ under some additional assumptions. The following lemma may be proved in an elementary way. Therefore its proof is omitted.

Lemma 1. Let $g \in C\left(\left[t_{0}-\tau, t_{0}\right)\right)$ and $\omega \in C(I)$. Then there is a unique function $\lambda=\lambda_{g}$ defined on $\left[t_{0}-\tau, \infty\right)$ by formulas (4), (5) which satisfies (3) on $I$. This function is continuous on $\left[t_{0}-\tau, \infty\right)$ if

$$
g^{(r)}\left(t_{0}-0\right)=g^{(r)}\left(t_{0}-\tau\right)+\omega^{(r)}\left(t_{0}\right)
$$

for $r=0$, and continuously differentiable on $\left[t_{0}-\tau, \infty\right)$ if, moreover, $g \in$ $C^{1}\left(\left[t_{0}-\tau, t_{0}\right)\right), \omega \in C^{1}(I)$, and (6) holds for $r=1$.

Lemma 2. Let $g \in C^{1}\left(\left[t_{0}-\tau, t_{0}\right)\right), g^{\prime}$ be nondecreasing on $\left[t_{0}-\tau, t_{0}\right)$, $\omega \in C^{1}(I)$ and $\omega^{\prime}$ be nondecreasing on I. Suppose, moreover, that (6) holds for $r=0$ and $r=1$. Then, for $t \in I$,

$$
\text { (7) } \begin{aligned}
{\left[g\left(t_{0}-0\right)+\right.} & \left.\frac{1}{\tau} \int_{t_{0}}^{t} \omega(s) d s\right]^{(s)} \\
& \leq \lambda_{g}^{(s)}(t) \leq\left[g\left(t_{0}-\tau\right)+\frac{1}{\tau} \int_{t_{0}}^{t+\tau} \omega(s) d s\right]^{(s)}, \quad s=0,1 .
\end{aligned}
$$

This follows from Lagrange's mean-value theorem.

Lemma 3. Suppose all assumptions of Lemma 2 are valid, $\omega(t) \geq q>0$, $q=$ const, on $I$ and $\lim _{t \rightarrow \infty} \omega(t+\tau) / \omega(t)=1$. Then

$$
\lambda_{g}(t) \sim \frac{1}{\tau} \int_{t_{0}}^{t} \omega(s) d s \quad \text { as } t \rightarrow \infty .
$$

The proof which is omitted uses the l'Hospital rule and inequalities (7) for $s=1$.

3. The main results. We will suppose that a solution of equation (1) has the form

$$
x(t)=\exp (-K \lambda(t))
$$


where $\lambda$ is a new unknown function and $0<K=$ const. The corresponding equation for $\lambda$ is

$$
\dot{\lambda}(t)=c(t) K^{-1} \exp \{K[\lambda(t)-\lambda(t-\tau)]\} .
$$

Equation (9) is in close connection with equation (3) for $\omega(t) \equiv$ $-K^{-1} \ln \left[c(t) K^{-1}\right]$, that is, with the equation

$$
\lambda(t)-\lambda(t-\tau)=-K^{-1} \ln \left[c(t) K^{-1}\right] .
$$

Theorem 1. Suppose $c \in C^{1}(I)$ and $g \in C^{1}\left(\left[t_{0}-\tau, t_{0}\right)\right)$ satisfy the following assumptions:

(i) $c$ is nonincreasing, $0<c(t)<K$ on $I$ where $K$ is some positive constant, and $c^{\prime} / c$ is nonincreasing on $I$;

(ii) $g(t)>0$ and $g^{\prime}$ is nondecreasing on $\left[t_{0}-\tau, t_{0}\right)$;

(iii) for $s \in\{0,1\}$,

$$
g^{(s)}\left(t_{0}-0\right)=g^{(s)}\left(t_{0}-\tau\right)-K^{-1}\left[\ln \left[c\left(t_{0}\right) K^{-1}\right]\right]^{(s)} .
$$

Moreover, suppose there are constants $M$ and $L, 0<M<L$, such that

(11) $M^{-1}\left[c(t) K^{-1}\right]^{1-M} \leq-(\tau K)^{-1} \ln \left[c(t) K^{-1}\right] \leq L^{-1}\left[c(t-\tau) K^{-1}\right]^{1-L}$

for $t \in I$ (left inequality) and for $t \in\left[t_{0}+\tau, \infty\right)$ (right inequality). Then there exists an uncountable set $\Lambda$ of functions $\lambda$ defined on $\left[t_{0}-\tau, \infty\right)$ and satisfying equation (9) on I. Moreover, for $\lambda \in \Lambda$,

$$
M \lambda_{g}(t)<\lambda(t)<L \lambda_{g}(t)
$$

for $t \in\left[t_{0}-\tau, \infty\right)$ where $\lambda_{g}$ is the solution of equation (10) defined by the initial function $g$.

Proof. We define $\Omega(t)$ where $t \in I$ to be the set of continuous functions $\varphi$ from $[t-\tau, t]$ into $\mathbb{R}$ such that

$$
M \lambda_{g}(t+\theta)<\varphi(t+\theta)<L \lambda_{g}(t+\theta)
$$

for all $\theta \in[-\tau, 0)$ and either $\varphi(t)=M \lambda_{g}(t)$ or $\varphi(t)=L \lambda_{g}(t)$. Further we define two functions $W_{1}(t, \lambda) \equiv \lambda-M \lambda_{g}(t)$ and $W_{2}(t, \lambda) \equiv \lambda-L \lambda_{g}(t)$ and we find the sign of derivatives of these functions along the solutions of equation (9) on the set $\Omega(t)$ for each $t \in I$. For $W_{2}(t, \lambda)$ and each $\varphi \in \Omega(t)$ such that $\varphi(t)=L \lambda_{g}(t)$ we obtain

$$
\begin{aligned}
\left.\frac{d W_{2}(t, \lambda)}{d t}\right|_{\lambda=\varphi(t)} & =\left.\left[c(t) K^{-1} \exp \{K[\lambda(t)-\lambda(t-\tau)]\}-L \lambda_{g}^{\prime}(t)\right]\right|_{\lambda=\varphi(t)} \\
& =c(t) K^{-1} \exp \{K[\varphi(t)-\varphi(t-\tau)]\}-L \lambda_{g}^{\prime}(t) .
\end{aligned}
$$

Using the right hand side inequality of (7) for $s=1$ where $\omega(t) \equiv-K^{-1}$ $\times \ln \left[c(t) K^{-1}\right]$ and the definition of $\Omega(t)$, we obtain 


$$
\begin{aligned}
\left.\frac{d W_{2}(t, \lambda)}{d t}\right|_{\lambda=\varphi(t)}> & c(t) K^{-1} \exp \left\{K L\left[\lambda_{g}(t)-\lambda_{g}(t-\tau)\right]\right\} \\
& +L(\tau K)^{-1} \ln \left[c(t+\tau) K^{-1}\right] \\
= & {\left[c(t) K^{-1}\right]^{1-L}+L(\tau K)^{-1} \ln \left[c(t+\tau) K^{-1}\right] . }
\end{aligned}
$$

Finally, in view of the right hand side inequality of (11), we conclude that

$$
\left.\frac{d W_{2}(t, \lambda)}{d t}\right|_{\lambda=\varphi(t)}>\left[c(t) K^{-1}\right]^{1-L}-\left[c(t) K^{-1}\right]^{1-L}=0 .
$$

For $W_{1}(t, \lambda)$ and each $\varphi \in \Omega(t)$ such that $\varphi(t)=M \lambda_{g}(t)$ we obtain by analogy

$$
\begin{aligned}
\left.\frac{d W_{1}(t, \lambda)}{d t}\right|_{\lambda=\varphi(t)}= & c(t) K^{-1} \exp \{K[\varphi(t)-\varphi(t-\tau)]\}-M \lambda_{g}^{\prime}(t) \\
< & c(t) K^{-1} \exp \left\{K M\left[\lambda_{g}(t)-\lambda_{g}(t-\tau)\right]\right\} \\
& +M(\tau K)^{-1} \ln \left[c(t) K^{-1}\right] \\
\leq & {\left[c(t) K^{-1}\right]^{1-M}-\left[c(t) K^{-1}\right]^{1-M}=0 }
\end{aligned}
$$

Now, by the topological method of T. Ważewski (see, for instance, [4], [9]) in the version of K. P. Rybakowski (e.g. [7], [8]) which is suitable for retarded functional differential equations, there is a set of functions $\Lambda$ such that if $\widetilde{\lambda} \in \Lambda$ then $M \lambda_{g}(t)<\widetilde{\lambda}(t)<L \lambda_{g}(t), t \in\left[t_{0}-\tau, \infty\right), \widetilde{\lambda}$ satisfies equation (13) on $I$ and the set $\left\{(t, \widetilde{\lambda}(t)): t=t_{0}, \widetilde{\lambda} \in \Lambda\right\}$ has the power of the continuum. That is, inequalities (12) hold and the conclusion of the theorem is true. The details of the application of the topological method are omitted because they can be found e.g. in [2], [4], [7-9]. The theorem is proved.

Remark 1. Using Lemmas 2 and 3 we may obtain concrete inequalities for solutions from $A$. E.g. if Theorem 1 holds, $c(t) K^{-1} \leq \nu<1, \nu=$ const on $I$ and

$$
\lim _{t \rightarrow \infty} \frac{\ln \left[c(t+\tau) K^{-1}\right]}{\ln \left[c(t) K^{-1}\right]}=1
$$

then we may (by Lemma 3) conclude that there are positive numbers $\varepsilon_{1}<1$ and $\varepsilon_{2}>1$ such that for each solution $\lambda \in \Lambda$ of equation (9),

$$
\frac{-\varepsilon_{1} M}{\tau K} \int_{t_{0}}^{t} \ln \left[c(s) K^{-1}\right] d s<\lambda(t)<\frac{-\varepsilon_{2} L}{\tau K} \int_{t_{0}}^{t} \ln \left[c(s) K^{-1}\right] d s
$$

as $t \rightarrow \infty$.

Remark 2. For a wide class of functions $c$ (e.g. $c(t)=\exp (-p t), 0<$ $p=$ const; $c(t)=\exp (-\exp (p t)), 0<p=$ const $)$ the constants $M$ and $L$ in 
Theorem 1 may be chosen such that $M \leq 1<L$.

Strictly speaking, we may expect the existence of solutions of equation (9) which satisfy inequalities similar to (13) without knowledge of the existence of the solution $\lambda=\lambda_{g}$ of equation (10). From this standpoint the statements of Lemmas $1-3$ can be used as a motivation for finding inequalities of some form for solutions of equation (9). Therefore the conditions of the following theorem are different from the assumptions of these lemmas.

THEOREM 2. Let $c \in C(I)$ be nonincreasing and such that $0<c(t)$ $<K$ on $I$ where $K$ is some positive constant. Moreover, suppose there are constants $M$ and $L, 0<M<L$, such that for $t \in I$,

$$
\begin{array}{r}
M^{-1} \exp \left\{\frac{-K M}{\tau} \int_{t+\tau}^{t+2 \tau} \ln \left[c(s) K^{-1}\right] d s\right\} \leq \frac{-K}{\tau c(t)} \ln \left[c(t+2 \tau) K^{-1}\right] \\
\leq L^{-1} \exp \left\{\frac{-K L}{\tau} \int_{t+\tau}^{t+2 \tau} \ln \left[c(s) K^{-1}\right] d s\right\}
\end{array}
$$

Then there exists an uncountable set $\Lambda_{1}$ of functions $\lambda$ defined on $\left[t_{0}-\tau, \infty\right)$ and satisfying equation (9) on I. Moreover, for $\lambda \in \Lambda_{1}$,

$$
\omega_{1}(t)<\lambda(t)<\omega_{2}(t)
$$

for $t \in\left[t_{0}-\tau, \infty\right)$, where

$$
\omega_{1}(t) \equiv \frac{-M}{\tau} \int_{t_{0}}^{t+2 \tau} \ln \left[c(s) K^{-1}\right] d s, \quad \omega_{2}(t) \equiv \frac{-L}{\tau} \int_{t_{0}}^{t+2 \tau} \ln \left[c(s) K^{-1}\right] d s .
$$

Proof. The proof is analogous to that of Theorem 1 and therefore we only describe the main points. Define $\Omega_{1}(t)$ where $t \in I$ to be the set of continuous functions $\varphi$ from $[t-\tau, t]$ into $\mathbb{R}$ such that $\omega_{1}(t+\theta)<\varphi(t+\theta)<$ $\omega_{2}(t+\theta)$ for all $\theta \in[-\tau, 0)$ and either $\varphi(t)=\omega_{1}(t)$ or $\varphi(t)=\omega_{2}(t)$. Further we define $W_{i}(t, \lambda) \equiv \lambda-\omega_{i}(t), i=1,2$. For $\varphi \in \Omega_{1}(t)$ with $\varphi(t)=\omega_{2}(t)$ we compute:

$$
\begin{aligned}
\frac{d W_{2}(t, \lambda)}{d t} & \left.\right|_{\lambda=\varphi(t)} \\
& =\left.\left[c(t) K^{-1} \exp \{K[\lambda(t)-\lambda(t-\tau)]\}+\frac{L}{\tau} \ln \left[c(t+2 \tau) K^{-1}\right]\right]\right|_{\lambda=\varphi(t)} \\
& >\frac{c(t)}{K} \exp \left\{K\left[\omega_{2}(t)-\omega_{2}(t-\tau)\right]\right\}+\frac{L}{\tau} \ln \left[c(t+2 \tau) K^{-1}\right] \\
& =\frac{c(t)}{K} \exp \left\{\frac{-K L}{\tau} \int_{t+\tau}^{t+2 \tau} \ln \left[c(s) K^{-1}\right] d s\right\}+\frac{L}{\tau} \ln \left[c(t+2 \tau) K^{-1}\right] \geq 0
\end{aligned}
$$


For $\varphi \in \Omega_{1}(t)$ with $\varphi(t)=\omega_{1}(t)$ we obtain

$$
\begin{aligned}
& \left.\frac{d W_{1}(t, \lambda)}{d t}\right|_{\lambda=\varphi(t)} \\
& =\left.\left[\frac{c(t)}{K} \exp \{K[\lambda(t)-\lambda(t-\tau)]\}+\frac{M}{\tau} \ln \left[c(t+2 \tau) K^{-1}\right]\right]\right|_{\lambda=\varphi(t)} \\
& <\frac{c(t)}{K} \exp \left\{K\left[\omega_{1}(t)-\omega_{1}(t-\tau)\right]\right\}+\frac{M}{\tau} \ln \left[c(t+2 \tau) K^{-1}\right] \\
& =\frac{c(t)}{K} \exp \left\{\frac{-K M}{\tau} \int_{t+\tau}^{t+2 \tau} \ln \left[c(s) K^{-1}\right] d s\right\}+\frac{M}{\tau} \ln \left[c(t+2 \tau) K^{-1}\right] \leq 0 .
\end{aligned}
$$

The theorem is proved.

For equation (1), as follows from Theorems 1 and 2 and from transformation (8), the following result holds:

Theorem 3. (a) Let all assumptions of Theorem 1 be valid. Then there exists an uncountable set $X$ of functions $x$ defined on $\left[t_{0}-\tau, \infty\right)$ and satisfying equation (1) on I such that

$$
\exp \left\{-L \lambda_{g}(t)\right\}<x(t)<\exp \left\{-M \lambda_{g}(t)\right\}
$$

for $t \in\left[t_{0}-\tau, \infty\right)$ where $\lambda_{g}$ is the solution of equation (10) defined by the initial function $g$.

(b) Let all assumptions of Theorem 2 be valid. Then there exists an uncountable set $X_{1}$ of functions $x$ defined on $\left[t_{0}-\tau, \infty\right)$ and satisfying equation (1) on I such that

$$
\exp \left\{-\omega_{2}(t)\right\}<x(t)<\exp \left\{-\omega_{1}(t)\right\}
$$

for $t \in I$.

By means of the transformation $x(t)=w(t) \exp \left\{\int_{t_{0}}^{t} a(s) d s\right\}$ equation (2) can be transformed into $\dot{w}(t)=-c_{1}(t) w(t-\tau)$ where $c_{1}(t) \equiv b(t)$ $\times \exp \left\{\int_{t}^{t-\tau} a(s) d s\right\}$. Since the latter equation has the form (1), the following result holds for equation (2):

Theorem 4. (a) Let all assumptions of Theorem 1 be valid with $c(t) \equiv$ $c_{1}(t)$. Then there exists an uncountable set $X_{2}$ of functions $x$ defined on $\left[t_{0}-\tau, \infty\right)$ and satisfying equation (2) on I and inequalities (16) on $\left[t_{0}-\tau, \infty\right)$ where $\lambda_{g}$ is the solution of equation (10) (with $\left.c(t) \equiv c_{1}(t)\right)$ defined by the initial function $g(t)$.

(b) Let all assumptions of Theorem 2 be valid with $c(t) \equiv c_{1}(t)$. Then there exists an uncountable set $X_{3}$ of functions $x$ defined on $\left[t_{0}-\tau, \infty\right)$ and satisfying equation (2) on I and inequalities (17) on $I$ (with $c(t) \equiv c_{1}(t)$ in the definitions (15) of $\left.\omega_{i}, i=1,2\right)$. 
EXAMPLE. The functions $x(t)=q \exp \left(-t^{2}\right), q=$ const, satisfy the equation

$$
\dot{x}(t)=-2 t[\exp (1-2 t)] x(t-1)
$$

which was used by Hale [3] as an illustration of the fact that solutions of nonautonomous linear homogeneous functional differential equations with bounded coefficients can be nonzero and approach zero more rapidly than each exponent.

If we put $c(t) \equiv 2 t \exp (1-2 t), \tau=2 M=K=1$ and $L=2$ in the formulation of Theorem $3(\mathrm{~b})$ then inequalities (14) hold for $t \in I$ if $t_{0}$ is sufficiently large. Thus for each $x \in X_{1}$ and large $t_{0}$,

$$
\exp \left(-2 t^{2}-10 t\right)<x(t)<\exp \left(-\frac{1}{2} t^{2}\right)
$$

for $t \in I$. This is in accordance with the above mentioned facts.

\section{References}

[1] R. Bellman and K. L. Cooke, Differential-Difference Equations, Academic Press, New York 1963.

[2] J. Diblík, On existence and asymptotic behaviour of solutions of singular Cauchy problem for certain system of ordinary differential equations, Fasc. Math. 18 (1988), $51-62$.

[3] J. Hale, Theory of Functional Differential Equations, Springer, New York 1977.

[4] Ph. Hartman, Ordinary Differential Equations, Wiley, New York 1964.

[5] V. B. Kolmanovskij and V. R. Nosov, Stability of Functional Differential Equations, Academic Press, 1986.

[6] V. Lakshmikantham and S. Leela, Differential and Integral Inequalities, Vol. 2, Academic Press, 1969.

[7] K. P. Rybakowski, Ważewski's principle for retarded functional differential equations, J. Differential Equations 36 (1980), 117-138.

[8] -, A topological principle for retarded functional differential equations of Carathéodory type, ibid. 39 (1981), 131-150.

[9] T. Ważewski, Sur un principe topologique de l'examen de l'allure asymptotique des intégrales des équations différentielles ordinaires, Ann. Soc. Polon. Math. 20 (1947), 279-313.

DEPARTMENT OF MATHEMATICS

FACULTY OF ELECTRICAL ENGINEERING

TECHNICAL UNIVERSITY OF BRNO

AREÁL VUT KRAVÍ HORA 21 (XV)

60200 BRNO, CZECH 